Instituto Internacional de Investigación y Desarrollo Tecnológico Educativo INDTEC, C.A.

DOI: https://doi.org/10.29394/Scientific.issn.2542-2987.2019.4.11.6.121-137

OAl-PMH: http://www.indteca.com/ojs/index.php/Revista Scientific/oai

Artículo Original / Original Article

\title{
Estrategias Ambientales para la Mejora de las Áreas Verdes en Ciudad Tavacare, Estado Barinas
}

Autor: Lenies Jesús Piña Retamoza

Universidad pedagógica experimental Libertador, UPEL

leniesjp@gmail.com

Barinas Venezuela

https://orcid.org/0000-0003-0683-405X

Resumen

Las áreas verdes son el punto de partida para un poblado, ya que estas representan la mayor depuración de cualquier hecho contaminante que suscite en el entorno, producto de las distintas causas entre las que se encuentra crecimiento demográfico, insuficiencia en programas ambientales, indiferencia humana ante los problemas del ambiente, entre otros; la cual incide en la formación integral del hombre, ya que este necesita equilibrio ambiental, es decir hombre-naturaleza. Por tanto, dicho comportamiento ha llevado a reducir y generar un deterioro ambiental. El objetivo principal presentado en este trabajo se enmarca en desarrollar estrategias ambientales para la mejora de las áreas verdes, del sector "C," Terraza Consejo Comunal 28 de julio en Ciudad Tavacare, ubicado en el Municipio Barinas, estado Barinas, la metodología utilizada es de índole cuantitativa, el tipo de investigación es proyecto factible con diseño de campo. El instrumento aplicado para la recolección de datos fue el cuestionario para obtención de los resultados. La población estudiada son 192 sujetos y la muestra quedó representada con 57 sujetos, por otro lado, la confiabilidad quedo demostrada luego de la tabulación del instrumento con un resultado de 0,92. En este sentido, la conclusión se abordó en función a los objetivos sugeridos en la investigación, pues la aplicación de técnicas ambientales contribuyen al mejoramiento, de las áreas verdes a corto plazo, tal es el caso que se presenta en el complejo urbanístico Ciudad Tavacare, donde las áreas verdes son un espacio que se han venido eliminando progresivamente, pues los habitantes del sector no realizan ninguna estrategias para mantenerlas, donde aportan beneficios para sus habitantes y visitantes tanto por la estética del lugar como el esparcimiento que brindan las áreas verdes.

Palabra clave: desarrollo sostenible; participación comunitaria; calidad de vida; educación ambiental.

Fecha de Recepción: 05-04-2018

Fecha de Aceptación: 23-08-2018 


\title{
Environmental Strategies for the Improvement of Green Areas in Tavacare City, Barinas State
}

\begin{abstract}
The green areas are the starting point for a town, since they represent the greatest purification of any pollutant that arouses in the environment, product of the different causes among which demographic growth is found, insufficiency in environmental programs, human indifference to environmental problems, among others; which affects the integral formation of man, since he needs environmental balance, that is, man-nature. Therefore, this behavior has led to reduce and generate environmental deterioration. The main objective presented in this work is framed in developing environmental strategies for the improvement of green areas, sector C, "Terrace Community Council July 28" in Ciudad Tavacare, located in the Municipality Barinas, Barinas State, the methodology used is of a quantitative nature, the type of research is feasible project with field design. The instrument applied for the data collection was the questionnaire to obtain the results. The studied population is 192 subjects and the sample was represented with 57 subjects, on the other hand, the reliability was demonstrated after the tabulation of the instrument with a result of 0.92 . In this sense, the conclusion was addressed according to the objectives suggested in the research, since the application of environmental techniques contribute to the improvement of green areas in the short term, such is the case that occurs in the Ciudad Tavacare urban development. where the green areas are a space that have been progressively eliminated, because the inhabitants of the sector do not carry out any strategies to maintain them, where they provide benefits for their inhabitants and visitors both for the aesthetics of the place and the relaxation provided by the green areas.

Keyword: sustainable development; community participation; quality of life; environmental education.
\end{abstract}

Date Received: 05-04-2018

Date Acceptance: 23-08-2018 


\section{Introducción}

La contaminación ambiental, va en crecimiento motivado a la complejidad del desarrollo que busca el ser humano tales como: la globalización, indiscriminado uso de la tecnológica, poco interés en creación y/o aplicación de programas ambientales, actitud negativa con respecto al cuidado del ambiente, entre otras. Es importante reconocer que el bienestar de los seres vivos está aunado al equilibrio ambiental entre hombrenaturaleza, por lo tanto, es imprescindible estar atentos a los cambios ocurridos en el entorno para el cuidado y preservación del ambiente, donde esto implica satisfacer necesidades, exigidas por la naturaleza misma del ser humano. En este sentido, se precisa desarrollar estrategias ambientales para la mejora de las áreas verdes, del sector "C", Terraza Consejo Comunal 28 de julio en Ciudad Tavacare, ubicado en el Municipio Barinas, estado Barinas, las cuales realzan el mejoramiento de las áreas verdes, como alternativa útil y eficaz para evitar la contaminación ambiental, pues la misma ayuda a la purificación del aire.

Bajo esta premisa, en Venezuela el crecimiento poblacional es notable por tanto, se requiere la construcción de viviendas como bienestar social y económico para la población y esto implica la reducción de áreas verdes para realizar prácticas de crecimiento y desarrollo, tal es el caso del estado Barinas, específicamente en la parroquia Alto Barinas, donde se llevó a cabo el complejo urbanístico llamado "Ciudad Tavacare" este contribuyó a la solución del problema de vivienda con la construcción de 5360 apartamentos aproximadamente, dividido en terrazas, a su vez divididos en bloque de 4 pisos, es decir dieciséis apartamento por bloque con una infraestructura, que incluye tres dormitorios, dos salas de baño, sala cocina, comedor $y$ estacionamiento, con pequeñas parcelas destinadas a las áreas verdes del conjunto residencial.

Aunque se puede observar, que existen terrazas (construcción de 4 
bloques) donde sus residentes le restan importancia ambiental encontrándose estas en total deterioro, en la que se puede mencionar el Consejo Comunal 28 de julio; consecuentemente, la construcción planificada de urbanismo es una forma de satisfacer la necesidades del hombre, pero se debe considerar las áreas verdes, las cuales son de carácter obligatorio en dicha construcciones, de allí pues, nace la inquietud de buscar estrategias ambientales como alternativa para el cuidado de estos espacios.

Es por ello, que la práctica de siembra de plantas contribuye al efecto positivo en el ambiente incidiendo en beneficios para los involucrados en dicha actividad, es decir la comunidad favorecida del sistema urbanístico, así que el hombre en su afán de buscar el bienestar ha encontrado oportunidades de vivir en común y con ello interactuar con la realidad social buscando soluciones viables que cubran sus expectativas. Tal es el caso de la plantación de árboles acorde al espacio urbanístico, los cuales brindan un sinfín de ventajas a la comunidad antes mencionada: ayudan a minimizar el deterioro ambiental, embellecen el entorno, participación comunitaria, entre otros.

\section{Bases Teóricas}

La fundamentación teórica plasmada en la siguiente investigación cumple un papel fundamental, pues la misma permite una clara y fácil comprensión del tema. En este apartado se busca la información teórica, pertinentes al estudio atendiendo la postura de diferentes autores y fuentes de información para concretar con precisión aspectos esenciales de las bases teóricas concerniente a la investigación, en tal sentido se presenta la siguiente teoría.

\subsection{Estrategias Ambientales}

Las estrategias representan habilidades que poseen los individuos para aplicarlas en un momento determinado, tal es el caso requerido para el 
desarrollo y aplicación de estrategias ambientales en tiempos cruciales que se vive en el entorno por el deterioro ambiental existente. En tal sentido, Rodríguez (s.f.), señala lo siguiente:

El termino Estrategias Ambiéntales es mencionado por primera vez en la Agenda 21 documento que fue suscripto en la Cumbre de la Tierra, la más vasta reunión de dirigentes mundiales, que se celebró en (el mes de junio) de 1992, en Río de Janeiro (Brasil) En donde se exhorta a los gobiernos a que adopten estrategias nacionales para el desarrollo sostenible. Estas deberán elaborarse con la amplia participación de todos los sectores, incluidas las organizaciones no gubernamentales y el público en general (pág. 2).

De allí pues, reconocer la importancia de donde proviene el término de estrategias ambientales avizora un panorama esperanzador, ya que el fin único de esta reunión y dejándolo así denotado en el documento es incentivar la población mundial que desde su realidad, aplique técnicas ambientales, las cuales contribuyen a minimizar el daño que se le ha venido ocasionando a la naturaleza y que ha ido creciendo, ya que las personas no aplican estrategia alguna para frenar esta situación, en dicho documento también se incita a los gobiernos que en su plan de estrategias nacionales, involucren al ambiente para motivar así a las personas a participar en ellas, logrando en conjunto un desarrollo sostenible.

\subsection{Importancia de las estrategias ambientales}

Entre los retos que se plantea el ser humano para alcanzar el máximo bienestar es tener presente el cuidado del ambiente, pues este representa el punto de partida para la conformación social que se encamina hacia el equilibrio ambiental pretendido para lograr la protección del entorno, para ello, debe coexistir un compromiso de participación masiva por parte de la comunidad que los haga conscientes del daño causado a la naturaleza, y así se pueda retribuir con acciones ambientalistas que mejoren la calidad de vida 
ambiental, también asumir con criterio las soluciones viables que contribuyan al desarrollo sostenible de la colectividad y a su vez sean modelo a nivel local, regional y nacional que tengan auge a nivel mundial, sin olvidar que se debe comenzar desde lo particular hacia la general, es decir, iniciar por resolver problemas ambientales desde el hogar hasta llegar aplicar técnicas funcionales a mayor alcance.

No se puede hablar de aplicación de estrategias ambientales sin hablar de educación ambiental, ya que es una forma de buscar soluciones a las problemáticas ambientales, ya que se abarca todo una comunidad completa, por ejemplo si se habla de educación ambiental formal, se hace referencia a la transversalidad inmersa en el diseño curricular; por ello, Tobasura y Sepúlveda (1997), citados por Fuentes, Caldera y Mendoza (2006): señalan "La Educación Ambiental no puede ser un curso aislado en los currículos de los diferentes niveles de la educación, sino un proceso sistémico y organizado que involucre todas las disciplinas y saberes existentes" (pág. 41).

De acuerdo a lo referido por los autores, indica la importancia que tiene la educación ambiental, en la educación formal en cada uno de sus niveles educativos, ya teniendo objetivos claros para impartir la educación ambiental, se obtendrán más fácil las estrategias de aplicación ante alguna problemática ambiental que se presente en un lugar determinado, por ello la preparación académica de los docentes es el punto de partida buenas estrategias afianzadas con su experiencia y el conocimiento que puedan tener y que a su vez se encargan de impartir a los estudiantes, y de aquí ellos llevan soluciones a las instituciones $u$ organismos principalmente.

\section{3. Áreas Verdes}

Las áreas verdes representan un significativo aporte a la calidad de vida de la población, pues su funcionamiento fortalece las ventajas de un ambiente libre de contaminación, de allí que las mismas requieren especial atención 
para su constitución que va desde la ubicación, especies y mantenimiento que necesitan en todos sus ámbitos. Al respecto, áreas verdes las señala Ojeda y Espejel (2014), citados por Márquez (2017), así:

Con una visión más amplia las áreas verdes están constituidas por todos aquellos parques, jardines, camellones, glorietas, áreas naturales y deportivas que forman parte de determinada ciudad, las cuales han pasado de ser elementos secundarios del paisaje, con fines solamente estéticos y recreativos, a convertirse en áreas de gran importancia, debido su funcionabilidad y a los beneficios ecológicos que aportan a la sociedad (pág. 234).

La cita antes expuesta, refiere a la identificación de los espacios verdes ubicados dentro de la ciudad, o una comunidad que puede estar rodeadas de plantas de tipo rastreras hasta árboles, es decir una combinación de plantas que hacen de aquel lugar llamativo, recibiendo el nombre de jardín, y que a su vez está aportando beneficios naturales, como respirar aire puro por los seres vivos que están alrededor del mismo, sirviendo también de área de relajación y esparcimiento a quienes interactúan en el lugar.

Consecuentemente, las áreas verdes están constituidas por plantaciones en la que se destacan árboles, arbustos, plantas rastreras siendo estas de cualquier especie, adaptando cada espacio a la necesidad requerida tales como, esparcimiento, recreación, ecología, protección, entre otros. Por ello la trascendencia de estas para que el hombre pueda vivir en armonía con la naturaleza sin ocasionar daño, de allí pues, los individuos deben involucrarse en el aspecto ambiental durante toda su formación académica incluyendo la temática de los espacios destinados para áreas verdes, por los grandes beneficios que aportan estos en la vida del hombre.

Aunque en realidad existen casos deplorables en cuanto a áreas verdes se refiere, pues muestran un escenario distinto al que pretende la educación formal, es decir, se encuentran deteriorados y/o destinados para otros fines. 
Por esta razón, es imprescindible la formación educativa ambiental que abarque todos los niveles y espacio de la colectividad (hogar, colegio, comunidad) y así se contribuye al logro de los objetivos orientados a revertir los daños ocasionados al ecosistema.

Por otro lado, no se puede aislar el rol que cumplen las industrias y empresas ya sean públicas o privadas que ocasionan grandes daños al ambiente y por ende las actividades productivas de estas, que se supone están normadas, por cuanto son funciones de las autoridades competentes en conjunto con el estado y las organizaciones ambientalistas, por ejemplo el Ministerio del Poder Popular para Ecosocialismo y Agua, trabajar de la mano para lograr el propósito de recuperar las áreas que alguna vez fueron espacios verdes de manera natural, por ello la interacción con la naturaleza forma parte del cuidado de la salud físico-mental del hombre, es decir, que las áreas verdes son parte de la calidad de vida requerida, pues fortalecen el bienestar de las personas, a través del contacto con los espacios verdes se ayuda a la salud en todos los sentidos particularmente a aquellas personas residentes en ciudades, pues es la forma de sentirse libre, relajados hasta el punto de ser recomendados como terapia en casos puntuales; de allí se conoce que la relación con espacios verdes y abiertos son ideales para reducir el estrés, pero cada quien asume la actitud conveniente hay libertad de decisión "Amar o destruir" y la intención es el amor por la naturaleza así como a las mascotas se le dan los cuidados necesarios, del mismo modo se debe hacer con la naturaleza, lo cual es garantía saludable en un mundo mejor para generaciones presentes y futuras.

\section{4. Áreas verdes en urbanismo}

Las áreas verdes en urbanismos son espacios utilizados para el esparcimiento, recreación que a su vez sirven para la ornamentación del mismo, donde dicho espacio es óptimo para el disfrute de todos los que habitan 
en el lugar, de allí, La Comisión Nacional de Medio Ambiente, citada por Del Pozo (2009), la define: "Como los espacios urbanos, o de periferia a éstos, predominantemente ocupados con árboles, arbustos o plantas, que pueden tener diferentes usos, ya sea cumplir funciones de esparcimiento, recreación, ecológicas, ornamentación, protección, recuperación y rehabilitación del entorno, o similares" (pág. 1).

De allí, la referencia del autor asevera el beneficio que trae consigo las áreas verdes particularmente en los urbanismos, pues ofrecen bienestar integral, ya que las plantaciones urbanas mejoran la calidad del aire.

Al respecto, Ciudad Tavacare, al igual que otro urbanismo en algún momento estuvo conformada por espacios verdes y antes de ser una zona urbana era una finca compuesta por árboles y pasto divididos en potreros, por lo tanto al empezar los movimientos y el replanteamiento del terreno desaparecen todas las áreas verdes trayendo consigo la deforestación total, una vez terminado el urbanismo siembran algunas plantas que por falta de mantenimiento se secaron y otras fueron arrancadas por las personas para colocarlas en los alrededores de sus áreas perimetrales.

Consecuentemente no quiere decir que Ciudad Tavacare no tenga áreas verdes, al contrario los residentes llevan la reforestación de esas áreas al igual que el sector "C" que cuenta con los mismos espacios que se han ido recuperando de manera voluntaria para darle valor agregado tal es el caso del sector "C" terraza consejo comunal 28 de julio, pero no existe una planificación de actividades y para ello es necesario crear un plan organizado que dé respuestas efectivas en cuanto a la recuperación y mantenimiento de los espacios con los cuales cuenta esta comunidad para las áreas verdes.

\section{Metodología}

La metodología utilizada intenta promover estrategias ambientales para la mejora de las áreas verdes, del sector "C", terraza consejo comunal 28 de 
julio, en Ciudad Tavacare, parroquia Alto Barinas, municipio Barinas, estado Barinas. El enfoque investigativo se orienta a investigación cuantitativa, la cual es señalada por Hurtado y Toro (2005), así:

Desde el punto de vista metodológico se suele denominar cuantitativa a la investigación que, predominantemente, tiende a usar instrumentos de medición y comparación que proporcionan datos cuyo estudio requiere el uso de modelos matemáticos y de la estadística. Por contraste se denomina cualitativas a las investigaciones que usan herramientas de obtención y manejo de información que no parten de la necesidad de utilizar las estadísticas o matemáticas para llegar a conclusiones (pág. 54).

Aunado al enfoque investigativo se tiene el tipo de investigación, la cual se determinó como proyectiva, donde Hurtado (2006): señala "intenta proponer soluciones a una situación determinada a partir de un proceso previo de indagación. Implica explorar, describir, explicar y proponer alternativas de cambio, mas no necesariamente ejecutar la propuesta" (pág. 117).

Consecuentemente, este tipo de investigación también es conocido como "proyecto factible", que de acuerdo a la Universidad Pedagógica Experimental Libertador (UPEL, 2012): consiste "en la investigación, elaboración y desarrollo de una propuesta de un modelo operativo viable para solucionar problemas, requerimiento o necesidades de organizaciones 0 grupos sociales" (pág. 21); en este sentido, la investigación presentada es una propuesta, la cual se ejecutó, en función a estrategias ambientales para la mejora de las áreas verdes, del sector "C", terraza Consejo Comunal 28 de julio en Ciudad Tavacare, estado Barinas; con diseño de campo, que Según Arias (2006a): la señala como "aquella que consiste en la recolección de todos directamente de los sujetos investigados, o de la realidad donde ocurren los hechos..." (pág. 31).

También, se tomó la población como aspecto resaltante para la investigación, donde la misma es señalada por Chávez (2001), como: "el 
universo de la investigación, sobre el cual se pretende generalizar resultado. Está constituida en características y sustratos que permiten distinguir los sujetos, unos de otros" (pág. 162); el estudio en cuestión del complejo urbanístico, Ciudad Tavacare, sector "C", terraza Consejo Comunal 28 de julio, donde está conformado por una población de 192 sujetos; para concretar la investigación era necesario recopilar información de fuente primaria, para ello se utilizó un instrumento denominado cuestionario, donde Arias (2006b): lo define como "modalidad de encuesta que se realiza de forma escrita mediante un instrumento en formato en papel contentivo de una serie de preguntas... y debe ser llenado por el encuestado, sin intervención del encuestador" (pág. 74).

Finalmente, se realiza el procesamiento de datos, los mismos se plasmaron en función de los objetivos, luego se calculó para hacer la agrupación y el análisis respectivo utilizando cuadros de frecuencia con respecto a las respuestas suministradas, Balestrini (2001): sugiere "a partir del análisis e interpretación de los resultados se intentará especificar y mostrar el conjunto de aspectos y propiedades que configuran el problema estudiado" (pág. 171). Para el análisis se presentan dos cuadros con sus respectivos criterios para poder desarrollar un plan estratégico para la mejora de áreas verdes en el sector antes mencionado, objeto de estudio.

Cuadro nro. 1: Existen condiciones físicas para las mejoras de áreas verdes en Ciudad Tavacare Sector "C"

\begin{tabular}{|c|c|c|c|c|c|c|}
\hline \multirow{2}{*}{ INDICADOR } & \multicolumn{2}{|c|}{ Si } & \multicolumn{2}{c|}{ No } & \multicolumn{2}{c|}{ TOTAL } \\
\cline { 2 - 7 } & Fa & $\%$ & Fa & $\%$ & Fa & Fr\% \\
\hline Suelo & $\mathbf{4 0}$ & $\mathbf{7 0}$ & $\mathbf{1 7}$ & $\mathbf{3 0}$ & $\mathbf{5 7}$ & $\mathbf{1 0 0}$ \\
\hline Superficie & $\mathbf{3 5}$ & $\mathbf{5 3}$ & $\mathbf{2 2}$ & $\mathbf{4 7}$ & $\mathbf{5 7}$ & $\mathbf{1 0 0}$ \\
\hline Ubicación & $\mathbf{5 0}$ & $\mathbf{8 8}$ & $\mathbf{7}$ & $\mathbf{1 2}$ & $\mathbf{5 7}$ & $\mathbf{1 0 0}$ \\
\hline Agua & $\mathbf{5 7}$ & $\mathbf{1 0 0}$ & $\mathbf{0}$ & $\mathbf{0}$ & $\mathbf{5 7}$ & $\mathbf{1 0 0}$ \\
\hline
\end{tabular}

Fuente: Datos tomados de las respuestas suministradas por los habitantes del sector "C" Ciudad Tavacare, estado Barinas; El Autor (2017). 
Con respecto a esta dimensión, la cual se ubica en las condiciones para las mejoras de las áreas verdes, se tienen los siguientes resultados de acuerdo con el cuestionario aplicado:

Suelo: El 70\% de los encuestados consideran que las condiciones del suelo sí influyen al momento de las mejoras de las áreas verdes, lo que indica hacer un análisis para conocer los nutrientes y tipo de suelo, para así determinar las especies a sembrar en el mejoramiento de las mencionadas áreas verdes, por otro lado el 30\% niega que las condiciones del suelo influyen para el mejoramiento de las áreas verdes, de allí la necesidad de dar orientaciones generales para demostrar que el tipo de suelo y por ende sus nutrientes son la clave para mantener las áreas verdes en excelente condiciones.

Superficie: la superficie destinada para las áreas verdes del urbanismo es considerada en un $35 \%$ acorde a lo que requiere el urbanismo, pues el espacio es suficiente para el cultivo y mantenimiento de estas destinadas al embellecimiento de la zona y por ende al cuidado del ambiente. Mientras que un $22 \%$ niega que la superficie no es la ideal, pues se requiere de espacios más amplios y acondicionados para el desarrollo y mantenimiento de áreas verdes.

Ubicación: en cuanto a el mencionado indicador, el $88 \%$ afirma que los espacios utilizados para las áreas verdes del urbanismo, cumplen con la expectativa, es decir se encuentran ubicadas al margen de los requerimientos habitacionales de allí parte el máximo aprovechamiento para mejorar dichas áreas, mientras que el $12 \%$ dice que no están bien ubicados, lo ideal sería darle forma y sentido con las variedades a cultivar y así proyectar la imagen del urbanismo.

Agua: este aspecto es otra de las condiciones inmersa para las mejoras de las áreas verdes, pues es un recurso indispensable para cualquier cultivo, es este caso de plantas ornamentales, de allí, se considera viable el desarrollo 
de la propuesta, ya que se cuenta con el mencionado recurso, lo que indica que las condiciones requeridas están dadas para poner en marcha el plan que permitirá accionar las estrategias ambientales efectivas en la solución de la problemática planteada.

Es preciso mencionar, que el recurso hídrico debe ser de fácil acceso, pues las personas comprometidas que en su momento le corresponda cumplir esta función dentro de las responsabilidades asumidas por cada uno de los actores sociales que habita en el sector " $C$ " de la ciudad Tavacare, para las mejoras de las áreas verdes y así evitar que se convierta en una limitante para llevar de manera efectiva el plan establecido.

Cuadro nro. 2: Factibilidad de un plan de estrategias ambientales en el mejoramiento de las áreas verdes.

\begin{tabular}{|c|c|c|c|c|c|c|}
\hline \multirow{2}{*}{ INDICADOR } & \multicolumn{2}{|c|}{ Si } & \multicolumn{2}{c|}{ No } & \multicolumn{2}{c|}{ TOTAL } \\
\cline { 2 - 7 } & $\mathbf{F a}$ & $\%$ & Fa & $\%$ & Fa & Fr\% \\
\hline Económica & $\mathbf{5 0}$ & $\mathbf{8 8}$ & $\mathbf{7}$ & $\mathbf{1 2}$ & $\mathbf{5 7}$ & $\mathbf{1 0 0}$ \\
\hline Técnica & $\mathbf{5 0}$ & $\mathbf{8 8}$ & $\mathbf{7}$ & $\mathbf{1 2}$ & $\mathbf{5 7}$ & $\mathbf{1 0 0}$ \\
\hline Social & $\mathbf{5 7}$ & $\mathbf{1 0 0}$ & $\mathbf{0}$ & $\mathbf{0}$ & $\mathbf{5 7}$ & $\mathbf{1 0 0}$ \\
\hline
\end{tabular}

Fuente: Datos tomados de las respuestas suministradas por los habitantes del sector "C" Ciudad Tavacare, estado, Barinas; El Autor (2017).

Uno de los requisitos que se necesitan para la aplicación de un plan estratégico es la factibilidad, el cual es imprescindible para el desarrollo de este, entre los aspectos para la factibilidad se encuentran los siguientes:

Económica: El 88\% de los encuestados afirman que económicamente si es factible aplicar un plan estratégico donde se aborden técnicas ambientales para las mejoras de áreas verdes en el urbanismo de Ciudad "Tavacare", mientras que el $12 \%$ niega la factibilidad económica para la aplicación de dicho plan, lo que se deduce que hay imitaciones por el alto costo inflacionario que circunda al país.

Sin embrago, es propio ante esta situación ser crítico, es decir, buscar 
alternativas donde se pueda aplicar un plan sin generar una inversión elevada, más aún que el beneficio incide de manera directa a la comunidad "Ciudad Tavacare" en este caso, donde el trabajo en equipo puede generar recursos propios para el mantenimiento y mejoras de las áreas verdes.

Técnica: En cuanto a la factibilidad técnica, el $88 \%$ afirmó que si es posible la aplicación de un plan estratégico pues, la tecnicidad puede ser abordada con instrumentos de fácil acceso y útiles para realizar siembra, poda y mantenimiento en general de las áreas verdes. Un $12 \%$ niega la factibilidad de la aplicación del plan sin el uso técnico como tal, sin embargo, se puede demostrar hay técnicas de fácil accesibilidad.

Social: el 100\% denomina que sí es factible la aplicación de las técnicas ambientales desde el punto de vista social, ya que hay valores sociales que predominan para el logro de objetivos, tal es el caso de las mejoras de las áreas verdes del sector " $\mathrm{C}$ " de Ciudad Tavacare. De esta manera, cuando la sociedad se organiza positivamente se beneficia la sociedad misma.

\section{Conclusiones}

El hombre a nivel mundial toma acciones de manera conscientes e inconscientes sobre las áreas verdes que redundan en su destrucción, sin embargo, hay quienes han hecho conciencia y están trabajando unidos por la causa ambiental, pues se han sumado para participar en programas, proyectos macros y micros con metas alcanzables en un plazo determinado y es imprescindible actuar ahora para un beneficio presente con miras al futuro pensando en el bienestar ambiental. Ciertamente, las plantas son el enlace para la obtención del oxígeno, componente vital para la sana y natural respiración de todo ser viviente.

Bajo esta premisa, la investigación planteada ratifica el beneficio que tienen las áreas verdes como parte de la calidad de vida para la sociedad, aunque conscientes de este factor aún se observa el poco accionar de algunas 
personas en sumarse al trabajo de equipo que implica el cuidado de las áreas verdes, insensiblemente no se percatan de la destrucción que se origina de manera progresiva. Por ello, es necesario estímulos internos y externos en las personas para abocarse a este llamado tal es el caso de los residentes del sector "C" de Ciudad Tavacare, específicamente en la terraza, consejo comunal 28 de julio, para acrecentar y fortalecer las áreas verdes, como valor agregado que contribuyen a la imagen corporativa del urbanismo y esparcimiento, tanto para quien vive allí, como quien lo visita, involucrando a niños y adultos, logrando satisfacción personal en aquellas personas que lo realizan y lo cuidan, puesto que con sus acciones están aportando la ayuda que tanto necesita la naturaleza.

Por ello, insistentemente la implementación de estrategias en función a una educación ambiental se orientó a concientización para así evitar que arrojen desperdicios en cualquier lugar, que en vez de caminar por la grama lo hagan por los espacios acondicionados para tal fin, sin olvidar el trabajo en equipo para el mantenimiento y cuidado de estas no basta la siembra sin control, sino mantener en el tiempo y espacio la actitud para el bienestar social desde un equilibrio ambiental.

\section{Referencias}

Arias, F. (2006a,b). El Proyecto de investigación. Introducción a la Metodología Científica. 5ta. Edición. Caracas, Venezuela: Editorial Espíteme, C.A.

Balestrini, M. (2001). Como se elabora el proyecto de investigación. 5ta Edición. Caracas, Venezuela: Consultores Asociados B.L.

Chávez N. (2001). Introducción a la Investigación Educativa, Maracaibo, Venezuela: Editorial La Columna.

Del Pozo, S. (2009). Definición de área verde. Chile: Universidad de Chile.

Recuperado de: 
http://arboriculturaurbana.blogspot.com/2009/01/definicin-de-rea-

verde.html

Fuentes, L., Caldera, Y., \& Mendoza I. (2006). La Transversalidad Curricular

y la Enseñanza de la Educación Ambiental. Revista ORBIS / Ciencias

Humanas, 2(4), 39-59, ISSN: 1856-1594. Recuperado de:

http://www.revistaorbis.org.ve/pdf/4/4Art2.pdf

Hurtado J. (2006). El proyecto de investigación. 4ta. Edición. Bogotá, Colombia: Editorial Quirón-Sypal.

Hurtado, I., \& Toro, G. (2005). Paradigmas y Métodos de Investigación en

Tiempos de Cambio. 5ta. edición. Valencia. Carabobo. Venezuela:

Edición Episteme Consultores Asociados C.A. Recuperado de:

https://epinvestsite.files.wordpress.com/2017/09/paradigmas-libro.pdf

Márquez, O. (2018). Jardines Ornamentales como Estrategia de

Participación local en el Embellecimiento de Áreas Verdes. Revista

Scientific, 3(7), 231-249. Recuperado de:

https://doi.org/10.29394/Scientific.issn.2542-2987.2018.3.7.12.231-249

Rodríguez L. (s.f.). La estrategia medioambiental y la formación del

profesional. Holguín, Cuba: Universidad de Holguín "Oscar Lucero

Moya". Recuperado de:

https://www.monografias.com/trabajos75/estrategia-medioambiental-

formacion-profesional/estrategia-medioambiental-formacion-

profesional2.shtml

UPEL (2012). Manual de Trabajo de Grado de Especialización y Maestría

y Tesis Doctorales. 4ta Edición. Caracas, Venezuela: Fondo Editorial de la Universidad Pedagógica Experimental Libertador, FEDUPEL. 


\section{Lenies Jesus Piña Retamoza \\ e-mail: leniesjp@gmail.com}

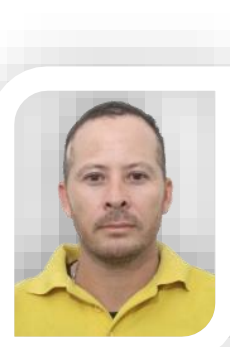

Nacido en Maracaibo, estado Zulia, Venezuela. Licenciado en Educación mención Geografía e Historia, en la Universidad Nacional Experimental de los Llanos Occidentales Ezequiel Zamora, UNELLEZ (2009), actualmente me desempeño, como Profesor en la Unidad Educativa Teresa de la Asunción, desde el año 2015.

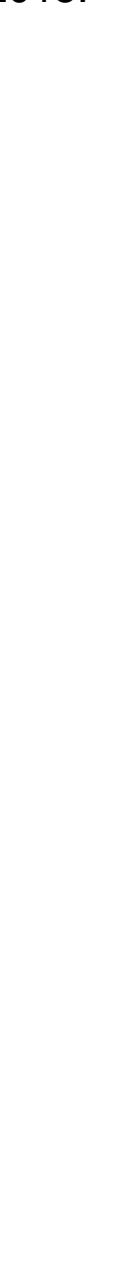

El contenido de este manuscrito se difunde bajo una Licencia de Creative Commons ReconocimientoNoComercial-Compartirlgual 4.0 Internacional 